\title{
Non-coplanar volumetric-modulated arc therapy (VMAT) for craniopharyngiomas reduces radiation doses to the bilateral hippocampus: a planning study comparing dynamic conformal arc therapy, coplanar VMAT, and non-coplanar VMAT
}

\author{
Megumi Uto, Takashi Mizowaki ${ }^{*}$, Kengo Ogura and Masahiro Hiraoka
}

\begin{abstract}
Background: Recent studies suggest that radiation-induced injuries to the hippocampus play important roles in compromising neurocognitive functioning for patients with brain tumors and it could be important to spare the hippocampus using modern planning methods for patients with craniopharyngiomas. As bilateral hippocampus are located on the same level as the planning target volume (PTV) in patients with craniopharyngioma, it seems possible to reduce doses to hippocampus using non-coplanar beams. While the use of non-coplanar beams in volumetric-modulated arc therapy (VMAT) of malignant intracranial tumors has recently been reported, no dosimetric comparison has yet been made between VMAT using non-coplanar arcs (ncVMAT) and VMAT employing only coplanar arcs (coVMAT) among patients with craniopharyngiomas. We performed a planning study comparing dose distributions to the PTV, hippocampus, and other organs at risk (OAR) of dynamic conformal arc therapy (DCAT), cOVMAT, and nCVMAT.

Methods: DCAT, coVMAT, and ncVMAT plans were created for 10 patients with craniopharyngiomas. The prescription dose was $52.2 \mathrm{~Gy}$ in 29 fractions, and $99 \%$ of each PTV was covered by $90 \%$ of the prescribed dose. The maximum dose was held below $107 \%$ of the prescribed dose. CoVMAT and ncVMAT plans were formulated to satisfy the following criteria: the doses to the hippocampus were minimized, and the doses to the OAR were similar to or lower than those of DCAT.

Results: The mean equivalent doses in 2-Gy fractions to $40 \%$ of the volumes of the bilateral hippocampus $\left[\mathrm{EQD}_{2}(40 \%\right.$ hippos $\left.)\right]$ were $15.4 / 10.8 / 6.5$ Gy for DCAT/coVMAT/ncVMAT, respectively. The $\mathrm{EQD}_{2}(40 \%$ hippos $)$ for ncVMAT were $<7.3 \mathrm{~Gy}$, which is the threshold predicting cognitive impairment, as defined by Gondi et al.. The mean doses to normal brain tissue and the conformity indices were similar for the three plans, and the homogeneity indices were significantly better for coVMAT and ncVMAT compared with DCAT.

(Continued on next page)
\end{abstract}

\footnotetext{
* Correspondence: mizo@kuhp.kyoto-u.ac.jp

Department of Radiation Oncology and Image-applied Therapy, Kyoto

University Graduate School of Medicine, 54 Shogoin Kawahara-cho, Sakyo-ku,

Kyoto 606-8507, Japan
}

\section{Biomed Central}

(c) 2016 The Author(s). Open Access This article is distributed under the terms of the Creative Commons Attribution 4.0 International License (http://creativecommons.org/licenses/by/4.0/), which permits unrestricted use, distribution, and reproduction in any medium, provided you give appropriate credit to the original author(s) and the source, provide a link to the Creative Commons license, and indicate if changes were made. The Creative Commons Public Domain Dedication waiver (http://creativecommons.org/publicdomain/zero/1.0/) applies to the data made available in this article, unless otherwise stated. 
(Continued from previous page)

Conclusions: NCVMAT is more appropriate than DCAT and coVMAT for patients with craniopharyngiomas. NcVMAT significantly reduces radiation doses to the bilateral hippocampus (to $50 \%$ that of the DCAT) without increasing the doses to normal brain tissue and other OAR.

Keywords: Hippocampus, Craniopharyngioma, Dosimetric comparison, Dynamic conformal arc therapy, Coplanar VMAT, Noncoplanar VMAT

\section{Background}

Patients with craniopharyngiomas exhibit a bimodal age distribution, and peaks are evident at ages 5-14 and 5074 years [1]. Aggressive surgery is often associated with increased frequencies of neurological, visual, cognitive, and neuroendocrinological side effects compared with limited surgery [2-4]. Therefore, limited surgery followed by radiotherapy (RT) is often used to manage craniopharyngiomas. This multidisciplinary approach (limited surgery and RT) affords a 10-year overall survival (OS) rate of 70-83\% and a 10-year progressionfree survival (PFS) rate of 60-69 \% [5-7].

As patients with craniopharyngiomas make good prognoses [5-7] and as pediatric patients seem to be more sensitive to radiation than adults, irradiation of normal tissue should be minimized. Cognitive decline is a recognized late effect of cranial irradiation, and it is suspected that radiation-induced injuries to the hippocampus are major contributors to neurocognitive deficits in patients with brain tumors [8-11]. The hippocampus is located close to the planning target volumes (PTVs) for craniopharyngiomas, and it could be important to spare the hippocampus using modern planning methods.

In terms of radiation techniques, 3D conformal external beam radiotherapy (3D-CRT) delivered using dynamic conformal arc therapy (DCAT), intensitymodulated radiotherapy (IMRT), and volumetricmodulated arc therapy (VMAT) are clinically employed to treat craniopharyngiomas [12]. In VMAT, both the shape of the radiation beam and the beam modulation can be changed while the gantry is rotating. VMAT reduces treatment delivery time and monitor units, and target coverage is equal to or better than that of IMRT [13]. VMAT can spare the hippocampus using inverse planning method but it remains unclear whether such sparing might increase the doses to other organs at risk (OAR). In addition, the use of non-coplanar beams in IMRT and VMAT for malignant intracranial tumors has recently been reported [14-16]. However, to the best of our knowledge, no dosimetric comparison has yet been made between VMAT using non-coplanar arcs (ncVMAT) and VMAT employing only coplanar arcs (coVMAT) for craniopharyngiomas with focus particularly on the hippocampal doses.
Therefore, we performed a planning study comparing the dose distributions to the PTVs and OAR of DCAT, coVMAT, and ncVMAT and identified the technique that maximally reduced doses to the hippocampus of patients with craniopharyngiomas.

\section{Methods}

This study followed all dictates of the Helsinki declaration and our Institutional Ethical Review Board approved the research (approval number E-1802).

\section{Patient population}

Ten patients with histologically confirmed craniopharyngiomas who were treated at our institution from November 2009 to November 2014 were included.

\section{Target and OAR delineation}

Contouring and treatment planning were performed using previously acquired computed tomography (CT) images and the Eclipse version 11.0.47 (Varian Medical Systems, Palo Alto, CA, USA). CT images $1.25-\mathrm{mm}$ thick were acquired by a Light Speed RT scanner (GE Healthcare, Milwaukee, WI, USA). Patients were immobilized in thermoplastic masks with bite blocks. Pre- and post-operative magnetic resonance imaging (MRI) scans were fused with the planning CT images.

As cystic lesions change in size during radiation therapy [17] and as residual tumors were possibly present, each clinical target volume (CTV) was defined to include any residual gross tumor and 5-mm thicknesses of any normal brain tissue attached to each tumor on preoperative MRI imaging. Then, the PTV was defined as the CTV plus 2-mm margin to allow for setup errors and patient motion. The lenses, eyes, optic nerves, chiasm, brainstem, hippocampus, and normal brain tissue were contoured as OAR. The hippocampus was delineated as described by Marsh et al. [18]. Couch structures were contoured and included in calculations.

\section{Treatment planning}

DCAT, coVMAT, and ncVMAT plans were created for each of 10 cases. Six-megavolt photon beams delivered by a Varian CL21iX linear accelerator through a Millennium 120-leaf multileaf collimator (Varian Medical 
Systems) were used in all plans. The Acuros XB dose Acurous calculation algorithm was employed; the calculation grid size was $2.5 \mathrm{~mm} \times 2.5 \mathrm{~mm}$. The dose prescribed for the PTV was 52.2 Gy in 29 fractions, and all plans were normalized to ensure that V90 $=99 \%$ (thus, $99 \%$ of the PTV was covered by $90 \%$ of the prescribed dose). The maximum doses to the brainstem, optic nerves/chiasm, and lens were set at less than 54, 55, and $10 \mathrm{~Gy}$, respectively.

\section{DCAT plans}

Each DCAT plan consisted of two coplanar and two non-coplanar arcs. We ensured that no beam irradiated the eyes. Two non-coplanar arcs were placed at couch angles of $45^{\circ}$ and $315^{\circ}$ (Fig. 1). All collimeter angles were set to $0^{\circ}$. The maximum dose (Dmax) was held below $107 \%$ of the prescribed dose.

\section{coVMAT plans}

Each coVMAT plan was created using the RapidArc system (Varian Medical Systems) and coplanar double arcs. One arc rotated clockwise from $181^{\circ}$ to $179^{\circ}$, and the collimator angle was $45^{\circ}$. The other arc rotated counterclockwise from $179^{\circ}$ to $181^{\circ}$, and the collimator angle was $315^{\circ}$. For both arcs, the couch positions were set to $0^{\circ}$, and avoidance sectors were placed to ensure that the eyes were not irradiated.

Dmax was set to be below 107 \% (as in DCAT planning). Optimization was performed until the final result met the following criteria: the doses to the eyes, lenses, optic nerves, chiasm, and brainstem were similar to or lower than those of DCAT and the dose to the hippocampus was maximally reduced.

\section{ncVMAT plans}

Each ncVMAT plan consisted of one coplanar arc and two non-coplanar arcs. We employed the RapidArc System. The coplanar arc was rotated clockwise with avoidances sectors not to irradiate the eyes. The couch positions and arc rotations of the two noncoplanar arcs were the same as those of DCAT. All collimater angles were set to $0^{\circ}$. Optimization was performed to ensure that the coVMAT criteria (see above) were met. As with the DCAT and coVMAT plans, Dmax was set to be below $107 \%$.

\section{Evaluation of treatment plans}

The DCAT, coVMAT, and ncVMAT plans were compared in terms of target homogeneity, target conformity, and the volumes of OAR irradiated. The homogeneity index (HI) was defined as (D2\% - D98\%)/D50\%, where D2\%, D98\%, and D50\% were the doses covering $2 \%$, $98 \%$, and $50 \%$ of the PTV, respectively. Two conformity indices (CIs) were calculated. One was the RTOG-CI, defined as $\mathrm{V}_{(90)} / \mathrm{V}_{\mathrm{PTV}}$, where $\mathrm{V}_{(90)}$ was the volume enclosed by $90 \%$ of the prescription isodose surface, and $\mathrm{V}_{\mathrm{PTV}}$ was the PTV. The other index was Ian Paddick's conformity index (IP-CI) described by Paddick et al.[19] and that was defined as $\mathrm{V}_{\mathrm{PTV}(90)}^{2} /\left(\mathrm{V}_{(90)} \times \mathrm{V}_{\mathrm{PTV}}\right)$. $\mathrm{V}_{\mathrm{PTV}(90)}$ is the volume of the PTV receiving $90 \%$ of the prescribed dose.

We calculated the equivalent doses in 2-Gy fractions to $40 \%$ of the bilateral hippocampus [EQD $2(40 \%$ hippos $)$ ]

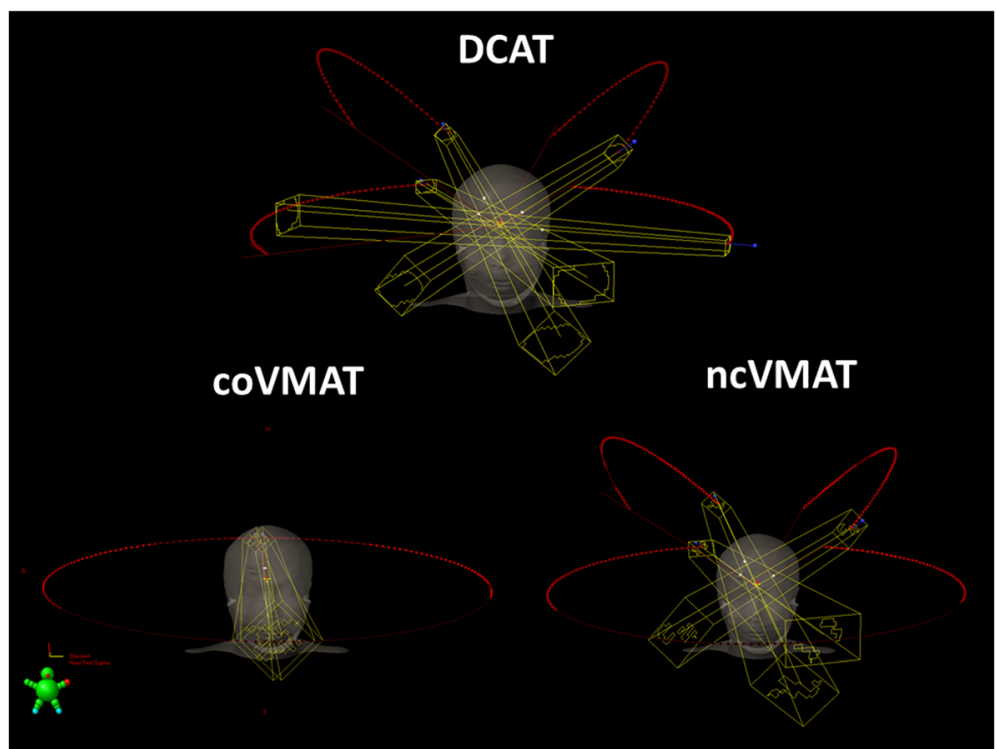

Fig. 1 Beam arrangement in a representative case; DCAT, coVMAT, and ncVMAT. DCAT = dynamic conformal arc therapy, coVMAT= coplanar volumetric-modulated arc therapy, ncVMAT = noncoplanar volumetric-modulated arc therapy 


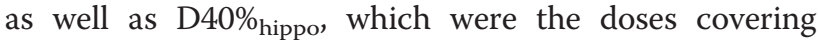
$40 \%$ of the volume of the bilateral hippocampus. The $\mathrm{EQD}_{2}(40 \%$ hippo $)$ index developed by Gondi et al. can be used to predict long-term neurocognitive functioning [9] and was derived from the $\mathrm{D} 40 \%_{\text {hippo, }}$, assuming an $\alpha / \beta$ ratio of 2 .

As the PTV varied individually, we also calculated normalized D40\% ${ }_{\text {hippo }}$ values (NV_D40\% $\%_{\text {hippos }}$ ). That of DCAT was set to unity. The NV_D40\% ${ }_{\text {hippo }}$ of coVMAT and ncVMAT were defined as D40\% ${ }_{\text {hippo }}$ (coVMAT)/ D $40 \%_{\text {hippo }}(\mathrm{DCAT})$ and $\mathrm{D} 40 \%_{\text {hippo }}$ (ncVMAT)/D40\% ${ }_{\text {hip- }}$ po (DCAT), respectively. We evaluated the doses to PTV and OAR using D2\% and D98\%.

\section{Statistical analysis}

All statistical analyses were performed with the aid of EZR (Saitama Medical Center, Jichi Medical University; http://www.jichi.ac.jp/saitama-sct/SaitamaHP.files/manual.html [20]), which is a graphical user interface for $\mathrm{R}$ (the R Foundation for Statistical Computing, Vienna, Austria, version 3.0.2). More precisely, EZR is a modified version of $\mathrm{R}$ commander version 1.24 , facilitating biostatistical evaluations.

Data from the three planning techniques (DCAT, coVMAT, and ncVMAT) were compared using two-way analysis of variance (ANOVA) across the entire cohort, followed by Bonferroni post hoc testing. A P-value $<0.05$ was considered to indicate statistical significance.

\section{Results}

\section{Target coverage}

The median PTV was $20.0 \mathrm{~cm}^{3}$ (range, $7.23-51.08 \mathrm{~cm}^{3}$ ). Table 1 summarizes the PTV index values. The HIs of coVMAT and ncVMAT were significantly better than those of DCAT $(\mathrm{p}<0.05)$. We found no significant difference among the three techniques in the RTOG-CI, IP-CI, or the mean PTV dose.

\section{Normal tissue doses}

Table 2 summarizes doses to the hippocampus and normal brain tissue. Mean doses to the bilateral hippocampus, $\mathrm{D} 40 \%_{\text {hippo }}$ and $\mathrm{EQD}_{2}\left(40 \%_{\text {hippo }}\right)$ emerged in the following order: ncVMAT, coVMAT, and DCAT. The mean $\mathrm{EQD}_{2}(40 \%$ hippo $)$ were $6.5,10.8$, and 15.4 Gy for ncVMAT, coVMAT, and DCAT, respectively. The mean $\mathrm{EQD}_{2}(40 \%$ hippo $)$ for ncVMAT were $<7.3 \mathrm{~Gy}$, which is the threshold dose to indicate cognitive impairment, as defined by Gondi et al. [9]. The NV_D40\% ${ }_{\text {hippo }}$ index tended to emerge in a similar manner and with statistical significance (ncVMAT: 0.4, coVMAT: 0.7, and DCAT: 1). The mean doses to normal brain tissue were similar in the three plans. Single coronal slices showing the dose distributions of each plan in a representative case are presented in Fig. 2.

The D2\% to both lenses were lower in the ncVMAT than in the other plans. There was no significant difference among the plans in the D2\% to the optic nerves.

Doses to OAR, excluding the hippocampus and normal brain tissue, are summarized in Table 3.

\section{Discussion}

We compared the dose distributions of DCAT, coVMAT, and ncVMAT plans for craniopharyngioma patients. NcVMAT delivered a significantly lower dose to the bilateral hippocampus than did the other two techniques; the mean $\mathrm{EQD}_{2}\left(40 \%_{\text {hippo }}\right)$ of ncVMAT was $<7.3 \mathrm{~Gy}$, which is the threshold predicting cognitive decline according to Gondi et al. [9]. NcVMAT achieved a better HI without increasing OAR doses. We thus found that ncVMAT was the best method to spare the bilateral hippocampus compared with DCAT and coVMAT.

Table 1 Summary of indices about the PTV

\begin{tabular}{llllllll}
\hline Index & $\begin{array}{l}\text { DCAT } \\
\text { (Mean } \pm \text { SD) }\end{array}$ & coVMAT & ncVMAT & $\begin{array}{l}\text { P-value } \\
\text { (ANOVA) }\end{array}$ & $\begin{array}{l}\text { P-value } \\
\text { (DCAT vs. coVMAT) }\end{array}$ & $\begin{array}{l}P \text {-value } \\
\text { (DCAT vs. ncVMAT) }\end{array}$ & $\begin{array}{l}P \text {-value } \\
\text { (coVMAT vs. ncVMAT) }\end{array}$ \\
\hline HI & $0.114 \pm 0.010$ & $0.103 \pm 0.008$ & $0.099 \pm 0.005$ & $<0.05$ & $<0.05$ & $<.05$ & 0.676 \\
RTOG-Cl & $1.758 \pm 0.672$ & $1.378 \pm 0.138$ & $1.429 \pm 0.175$ & 0.112 & & & 1 \\
IP-Cl & $0.615 \pm 0.164$ & $0.717 \pm 0.069$ & $0.694 \pm 0.078$ & 0.123 & & $<0.05$ & \\
D2\% (Gy) & $54.0 \pm 0.6$ & $53.1 \pm 0.6$ & $52.9 \pm 0.5$ & $<0.05$ & $<0.05$ & & \\
D98\% (Gy) & $48.0 \pm 0.2$ & $47.8 \pm 0.2$ & $47.8 \pm 0.2$ & 0.101 & & \\
Dmean (Gy) & $52.3 \pm 0.4$ & $51.7 \pm 1.3$ & $51.4 \pm 0.5$ & 0.077 & & & \\
\hline
\end{tabular}

If a significant difference was evident when data from the entire cohort were compared via two-way analysis of variance (ANOVA), the Bonferroni post hoc test was performed to compare pairs of modalities

PTV planning target volume, DCAT dynamic conformal arc therapy, coVMAT coplanar volumetric-modulated arc therapy, ncVMAT non-coplanar volumetricmodulated arc therapy

$\mathrm{HI}$ homogeneity index, $\mathrm{Cl}$ conformity index, $R T O G-\mathrm{Cl} \mathrm{Cl}$ as defined by the Radiation Therapy Oncology Group (RTOG), IP-Cl Cl as defined by Paddick et al. [19], D2\% dose to $2 \%$ of the volume, $D 98 \%$ dose to $98 \%$ of the volume, Dmean mean dose 
Table 2 Summary of doses delivered to the hippocampus and normal brain, in Gy

\begin{tabular}{llllllll}
\hline Structure/index & $\begin{array}{l}\text { DCAT } \\
\text { (Mean } \pm \text { SD) }\end{array}$ & coVMAT & ncVMAT & $\begin{array}{l}\text { P-value } \\
\text { (ANOVA) }\end{array}$ & $\begin{array}{l}\text { P-value } \\
\text { (DCAT vs. coVMAT) }\end{array}$ & $\begin{array}{l}\text { P-value } \\
\text { (DCAT vs. ncVMAT) }\end{array}$ & $\begin{array}{l}\text { P-value } \\
\text { (coVMAT vs. ncVMAT) }\end{array}$ \\
\hline Bilateral Hippo & & & & & & & \\
D2\% & $36.5 \pm 11.2$ & $29.5 \pm 12.6$ & $23.1 \pm 15.5$ & 0.095 & & $<0.05$ & 0.530 \\
Mean dose & $20.7 \pm 7.6$ & $15.4 \pm 8.1$ & $10.7 \pm 8.2$ & $<0.05$ & 0.515 & $<0.05$ & 0.326 \\
D40\% hippo $_{\text {EQD }}(40 \%$ hippo & $21.7 \pm 8.4$ & $16.2 \pm 7.6$ & $10.3 \pm 7.9$ & $<0.05$ & 0.406 & $<0.05$ & 0.51 \\
NV_D40\%hippo & $15.5 \pm 7.9$ & $10.8 \pm 6.3$ & $6.5 \pm 6.0$ & $<0.05$ & 0.41 & $<0.05$ & $<0.05$ \\
Normal brain & & $0.7 \pm 0.2$ & $0.4 \pm 0.2$ & $<0.05$ & $<0.05$ & & \\
Mean dose & $6.8 \pm 1.7$ & $6.2 \pm 1.5$ & $6.8 \pm 1.4$ & 0.563 & & & \\
\hline
\end{tabular}

If a significant difference was evident when data from the entire cohort were compared via two-way analysis of variance (ANOVA), the Bonferroni post hoc test was performed to compare pairs of modalities

DCAT dynamic conformal arc therapy, coVMAT coplanar volumetric-modulated arc therapy, ncVMAT non-coplanar volumetric-modulated arc therapy, SD standard deviation, Hippo hippocampus

D2\% dose to $2 \%$ of the volume, $D 40 \%$ hippo dose to $40 \%$ of the volume of the bilateral hippocampus

$E Q D_{2}(40 \%$ hippo) equivalent dose in 2-Gy fractions (assuming $\alpha / \beta=2$ ) to $40 \%$ of volume of the bilateral hippocampus

NV_D40\% hippo normalized value of D40\% hippo (the DCAT value was set to unity). The normalized values for coVMAT and ncVMAT were calculated as D $40 \%_{\text {hippo }}\left(\right.$ CoVMAT)/D40\% ${ }_{\text {hippo }}(\mathrm{DCAT})$ and D40\% ${ }_{\text {hippo }}(\mathrm{ncVMAT}) / \mathrm{D} 40 \%_{\text {hippo }}(\mathrm{DCAT})$, respectively

Neurocognitive toxicity after cranial irradiation is often seen in patients who received whole brain radiotherapy (WBRT) to treat metastatic brain tumors $[11,21]$. Many reports have explored the mechanism underpinning this phenomenon, and the dose to the temporal lobes seemed to predict the decline [22, 23]. Among temporal lobes, the hippocampus is considered important in terms of learning ability and memory functioning after irradiation $[8,10,21,24,25]$. The hippocampus are located in the median-temporal lobes, and Gondi et al. suggested that the hippocampal doses predicted impairments in the neurocognitive functioning of patients with benign or low-grade brain tumors [9]. A dose-response relationship seemed to be evident between the $\mathrm{EQD}_{2}(40 \%$ hippo $)$ and the impairment in Wechsler Memory Scale-III Word List. Word recall was delayed 18 months after irradiation; the $\mathrm{EQD}_{2}\left(40 \%{ }_{\text {hippo }}\right)$ threshold predicting cognitive impairment was $7.3 \mathrm{~Gy}$.

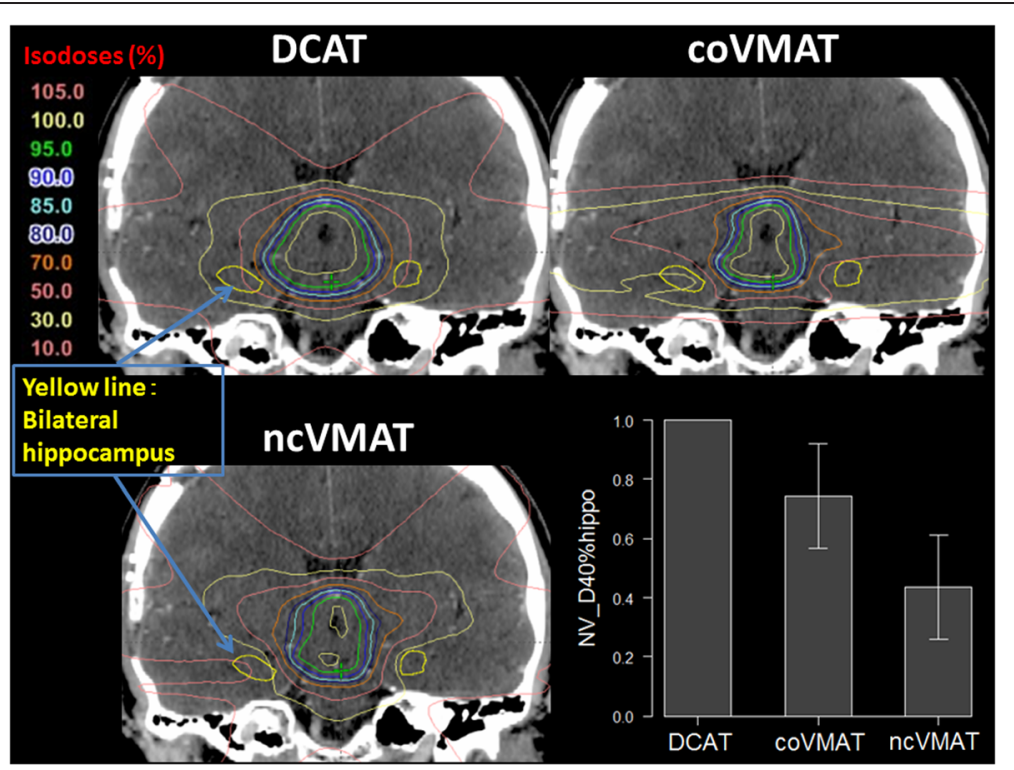

Fig. 2 Coronal plains of dose distributions in a representative case and a comparison of the normalized doses covering $40 \%$ of the volume of the bilateral hippocampus using DCAT, coVMAT, and ncVMAT. The yellow line shows the contour of the bilateral hippocampus. The normalized value of D40\% hippo indicates that the dose covering $40 \%$ of the volume of the bilateral hippocampus was significantly reduced in the following order: nCVMAT, coVMAT, and DCAT (ncVMAT 0.4, coVMAT 0.7, and DCAT 1). DCAT = dynamic conformal arc therapy, coVMAT = coplanar volumetric-modulated arc therapy, ncVMAT $=$ non-coplanar volumetric-modulated arc therapy, NV_D40\% $\%_{\text {hippo }}=$ normalized value of D40\% ${ }_{\text {hippo }}$ 
Table 3 Summary of OAR doses (hippocampus and normal brain tissue excluded)

\begin{tabular}{|c|c|c|c|c|c|c|c|}
\hline $\begin{array}{l}\text { Structure/ } \\
\text { index }\end{array}$ & $\begin{array}{l}\text { DCAT } \\
(\text { Mean } \pm \text { SD) }\end{array}$ & coVMAT & nCVMAT & $\begin{array}{l}P \text {-value } \\
\text { (ANOVA) }\end{array}$ & $\begin{array}{l}P \text {-value } \\
\text { (DCAT vs. coVMAT) }\end{array}$ & $\begin{array}{l}P \text {-value } \\
\text { (DCAT vs. ncVMAT) }\end{array}$ & $\begin{array}{l}P \text {-value } \\
\text { (coVMAT vs. ncVMAT) }\end{array}$ \\
\hline \multicolumn{8}{|c|}{ Lt. optic nerve } \\
\hline D2\% & $38.4 \pm 11.9$ & $37.2 \pm 11.2$ & $36.2 \pm 12.8$ & 0.915 & & & \\
\hline \multicolumn{8}{|c|}{ Rt. optic nerve } \\
\hline D2\% & $43.6 \pm 9.5$ & $40.5 \pm 10.9$ & $41.4 \pm 10.5$ & 0.789 & & & \\
\hline \multicolumn{8}{|l|}{ Chiasm } \\
\hline D2\% & $53.3 \pm 0.8$ & $52.2 \pm 0.9$ & $52.4 \pm 0.7$ & $<0.05$ & $<0.05$ & $<0.05$ & 1 \\
\hline \multicolumn{8}{|l|}{ Lt. lens } \\
\hline D2\% & $3.2 \pm 0.7$ & $2.2 \pm 0.7$ & $1.9 \pm 0.4$ & $<0.05$ & $<0.05$ & $<0.05$ & 0.854 \\
\hline \multicolumn{8}{|l|}{ Rt. lens } \\
\hline D2\% & $3.2 \pm 0.7$ & $2.3 \pm 0.7$ & $1.8 \pm 0.5$ & $<0.05$ & $<0.05$ & $<0.05$ & 0.224 \\
\hline
\end{tabular}

If a significant difference was evident when data from the entire cohort were compared via two-way analysis of variance (ANOVA), the Bonferroni post hoc test was performed to compare pairs of modalities

D2\% dose to $2 \%$ of the volume

SD standard deviation, DCAT dynamic conformal arc therapy, coVMAT coplanar volumetric-modulated arc therapy, ncVMAT noncoplanar volumetric-modulated arc therapy, Lt. left, Rt. right

Neurocognitive decline is often seen in patients with craniopharyngiomas, especially pediatric patients. Greenfield et al. found that $33 \%$ of pediatric patients who underwent surgery and IMRT exhibited neurocognitive and behavioral deficits at the last follow up and reported that a larger PTV was significantly associated with development of neurocognitive problems [5]. The greater the PTV, the closer the bilateral hippocampus was to that PTV; thus, the doses to the hippocampus may increase linearly with a rise in PTV. Therefore, hippocampal irradiation may contribute to cognitive decline in patients with craniopharyngiomas as well as other intracranial tumors, and it would be better to minimize doses to the hippocampus.

3D-CRT and VMAT are used to treat craniopharyngiomas in clinical practice, but the optimal method remains unknown. About $75 \%$ of craniopharyngiomas develop in the suprasellar region, close to the bilateral hippocampus, and it is difficult to set the $\mathrm{EQD}_{2}\left(40 \%_{\text {hippo }}\right)$ below 7.3 Gy when DCAT is planned [1]. VMAT can spare the hippocampus using an inverse planning method. In 2009, Wiggenraad et al. compared DCAT and noncoplanar IMRT (ncIMRT) in patients with various intracranial tumors [26]. When target conformity, homogeneity, and doses to the optic nerves and chiasm were all considered, the cited authors concluded that DCAT was equal to or better than ncIMRT in six of seven patients with skull-base meningiomas. The locations of such meningiomas are similar to those of craniopharyngiomas, and we found that ncVMAT afforded a better HI than and a similar CI to DCAT while providing equal or lower doses to other OAR. The reason for the betweenstudy difference is not clear, but it is possible that the use of VMAT rather than IMRT, and the method of optimization, are important to achieve better dose distribution.

We found that the HI of ncVMAT was significantly better than that of DCAT. Although statistical significance was lacking, the HI of ncVMAT was slightly better than that of coVMAT. It is well known that craniopharyngiomas undergo transient enlargement during radiation therapy [17]. The optic nerves and the chiasm are close to the PTV, and the HI is thus important in craniopharyngioma patients. If the target homogeneity is to be prioritized, ncVMAT may be more appropriate than DCAT or CoVMAT.

Noncoplanar arcs are usually used in DCAT to improve PTV conformity and homogeneity [12]. Recently, non-coplanar beams in IMRT and VMAT have been found to be useful for treating intracranial malignant tumors [14-16]. A study on the utility of non-coplanar beams in IMRT and VMAT for the treatment frontotemporal high-grade gliomas found that such beams reduced the doses to contralateral OAR, including the anterior/temporal lobes and optic structures [16]. The structures thus spared were usually coplanar and close to the PTVs of patients with fronto-temporal high-grade gliomas. The hippocampus is also located on the same level as the PTV in patients with craniopharyngiomas, and it appears that the use of ncVMAT compared with coVMAT may reduce the doses to coplanar structures. To the best of our knowledge, this is the first report to declare the utility of ncVMAT in terms of dose reductions to the hippocampus of patients with craniopharyngiomas.

One limitation of this study must be mentioned. This planning study compared dose distributions during DCAT, coVMAT, and ncVMAT, but it is unclear 
whether reducing the dose to the hippocampus actually assists in the preservation of cognitive functioning in clinical situations. Indeed, other factors contribute to the development of neurocognitive disorders in patients with irradiated craniopharyngiomas. These include the tumor per se, surgery, doses to the temporal lobes, shunt placement, presence of an Ommaya reservoir, diabetes insipidus, and low pre-irradiation growth hormone levels $[5,23,27,28]$. Prospective clinical trials on craniopharyngioma patients are required to explore whether the dosimetric advantage described herein affords real clinical benefits.

\section{Conclusions}

NcVMAT is more appropriate than DCAT and coVMAT for patients with craniopharyngiomas. NcVMAT significantly reduces the dose to the bilateral hippocampus (to $50 \%$ that of the dose for DCAT) without increasing the doses to normal brain tissue and other OAR. NcVMAT also improves target homogeneity.

\section{Availability of data and materials}

The dataset supporting the conclusions of this article is included within the supplementary material (Additional file 1).

\section{Ethical approval and consent to participate}

This study followed all dictates of the Helsinki Declaration and the ethical review board of Kyoto University Hospital and Faculty of Medicine approved the research (approval number E-1802). Written consent to participate was obtained from the patient.

\section{Consent to publish}

Written consent was obtained from the patient for publication of this report and any accompanying images.

\section{Additional file}

Additional file 1: The dataset supporting our findings. (XLSX $26 \mathrm{~kb}$ )

\section{Abbreviations}

3D-CRT, 3D conformal external beam radiotherapy; Cl, conformity index; coVMAT, volumetric-modulated arc therapy employing only coplanar arcs; CT, computed tomography; CTV, clinical target volume; D2\%, dose to $2 \%$ of

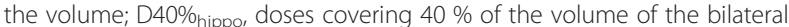
hippocampus; Dmax, maximum dose; $\mathrm{EQD}_{2}(40 \%$ hippos), equivalent doses in 2-Gy fractions to $40 \%$ of the volumes of the bilateral hippocampus; $\mathrm{HI}$, homogeneity index; IMRT, intensity-modulated radiotherapy; MRI, magnetic resonance imaging; ncIMRT, non-coplanar intensity-modulated radiotherapy; ncVMAT, volumetric-modulated arc therapy using non-coplanar arcs; NV_D40\% ${ }_{\text {hippos, }}$ normalized values of doses covering $40 \%$ of the volume of the bilateral hippocampus; OAR, organs at risk; OS, overall survival; PFS, progression-free survival; PTV, planning target volume; RT, radiotherapy; $\mathrm{V}_{(90) \text {, }}$ volume enclosed by $90 \%$ of the prescription isodose surface ; VMAT, volumetric-modulated arc therapy; $V_{\text {PTV }}(90)$, volume of the planning target volume receiving $90 \%$ of the prescribed dose; $V_{\text {PTV }}$, planning target volume; WBRT, whole brain radiotherapy.

\section{Competing interests}

The authors declare that they have no competing interests.

\section{Authors' contributions}

$\mathrm{MU}$ performed the planning study and the statistical analysis, and drafted the manuscript. TM, KO and MH conceived of the study, and participated in its design and coordination, and helped to draft the manuscript. All authors read and approved the final manuscript.

\section{Acknowledgements \\ None.}

\section{Funding}

This work was funded by JSPS KAKENHI Grant Number $15 \mathrm{~K} 18444$ and the [Practical Research for Innovative Cancer Control (16ck0106151h0002)] from the Japan Agency for Medical Research and development, AMED.

Received: 8 March 2016 Accepted: 14 June 2016

Published online: 23 June 2016

References

1. Jane Jr JA, Laws ER. Craniopharyngioma. Pituitary. 2006;9:323-6.

2. Clark AJ, Cage TA, Aranda D, Parsa AT, Auguste Kl, Gupta N. Treatmentrelated morbidity and the management of pediatric craniopharyngioma. J Neurosurg Pediatr. 2012;10:293-301.

3. Ali ZS, Bailey RL, Daniels LB, Vakhshori V, Lewis DJ, Hossain AT, et al. Comparative effectiveness of treatment options for pediatric craniopharyngiomas. J Neurosurg Pediatr. 2014;13:178-88.

4. Sughrue ME, Yang I, Kane AJ, Fang S, Clark AJ, Aranda D, et al. Endocrinologic, neurologic, and visual morbidity after treatment for craniopharyngioma. J Neurooncol. 2011;101:463-76.

5. Greenfield BJ, Okcu MF, Baxter PA, Chintagumpala M, Teh BS, Dauser RC, et al. Long-term disease control and toxicity outcomes following surgery and intensity modulated radiation therapy (IMRT) in pediatric craniopharyngioma. Radiother Oncol. 2015;114:224-9.

6. Masson-Cote L, Masucci GL, Atenafu EG, Millar BA, Cusimano M, Croul S, et al. Long-term outcomes for adult craniopharyngioma following radiation therapy. Acta Oncol. 2013;52:153-8.

7. Harrabi SB, Adeberg S, Welzel T, Rieken S, Habermehl D, Debus J, et al. Long term results after fractionated stereotactic radiotherapy (FSRT) in patients with craniopharyngioma: maximal tumor control with minimal side effects. Radiat Oncol. 2014;9:203.

8. Chang EL, Wefel JS, Hess KR, Allen PK, Lang FF, Kornguth DG, et al. Neurocognition in patients with brain metastases treated with radiosurgery or radiosurgery plus whole-brain irradiation: a randomised controlled trial. Lancet Oncol. 2009;10:1037-44.

9. Gondi V, Hermann BP, Mehta MP, Tome WA. Hippocampal dosimetry predicts neurocognitive function impairment after fractionated stereotactic radiotherapy for benign or low-grade adult brain tumors. Int J Radiat Oncol Biol Phys. 2013;85:348-54.

10. Gondi V, Pugh SL, Tome WA, Caine C, Corn B, Kanner A, et al. Preservation of memory with conformal avoidance of the hippocampal neural stem-cell compartment during whole-brain radiotherapy for brain metastases (RTOG 0933): a phase II multi-institutional trial. J Clin Oncol. 2014;32:3810-6.

11. Khuntia D, Brown P, Li J, Mehta MP. Whole-brain radiotherapy in the management of brain metastasis. J Clin Oncol. 2006;24:1295-304.

12. Aggarwal A, Fersht N, Brada M. Radiotherapy for craniopharyngioma. Pituitary. 2013;16:26-33.

13. Jeong Y, Lee SW, Kwak J, Cho I, Yoon SM, Kim JH, et al. A dosimetric comparison of volumetric modulated arc therapy (VMAT) and non-coplanar intensity modulated radiotherapy (IMRT) for nasal cavity and paranasal sinus cancer. Radiat Oncol. 2014;9:193.

14. Audet C, Poffenbarger BA, Chang P, Jackson PS, Lundahl RE, Ryu SI, et al. Evaluation of volumetric modulated arc therapy for cranial radiosurgery using multiple noncoplanar arcs. Med Phys. 2011;38:5863-72

15. Beltran C, Gray J, Merchant TE. Intensity-modulated arc therapy for pediatric posterior fossa tumors. Int J Radiat Oncol Biol Phys. 2012;82:e299-304.

16. Panet-Raymond V, Ansbacher W, Zavgorodni S, Bendorffe B, Nichol A, Truong PT, et al. Coplanar versus noncoplanar intensity-modulated radiation therapy (IMRT) and volumetric-modulated arc therapy (VMAT) treatment 
planning for fronto-temporal high-grade glioma. J Appl Clin Med Phys. 2012;13:3826.

17. Beltran C, Naik M, Merchant TE. Dosimetric effect of target expansion and setup uncertainty during radiation therapy in pediatric craniopharyngioma. Radiother Oncol. 2010;97:399-403.

18. Marsh JC, Gielda BT, Herskovic AM, Abrams RA. Cognitive sparing during the administration of whole brain radiotherapy and prophylactic cranial irradiation: current concepts and approaches. J Oncol. 2010;2010:198208.

19. Paddick I. A simple scoring ratio to index the conformity of radiosurgical treatment plans. Technical note. J Neurosurg. 2000;93 Suppl 3:219-22.

20. Kanda Y. Investigation of the freely available easy-to-use software 'EZR' for medical statistics. Bone Marrow Transplant. 2013;48:452-8.

21. Sun A, Bae K, Gore EM, Movsas B, Wong SJ, Meyers CA, et al. Phase III trial of prophylactic cranial irradiation compared with observation in patients with locally advanced non-small-cell lung cancer: neurocognitive and quality-oflife analysis. J Clin Oncol. 2011;29:279-86.

22. Jalali R, Mallick I, Dutta D, Goswami S, Gupta T, Munshi A, et al. Factors influencing neurocognitive outcomes in young patients with benign and low-grade brain tumors treated with stereotactic conformal radiotherapy. Int J Radiat Oncol Biol Phys. 2010;77:974-9.

23. Merchant TE, Kiehna EN, Kun LE, Mulhern RK, Li C, Xiong X, et al. Phase II trial of conformal radiation therapy for pediatric patients with craniopharyngioma and correlation of surgical factors and radiation dosimetry with change in cognitive function. J Neurosurg. 2006;104:94-102.

24. Monje ML, Palmer T. Radiation injury and neurogenesis. Curr Opin Neurol. 2003;16:129-34.

25. Redmond KJ, Mahone EM, Terezakis S, Ishaq O, Ford E, McNutt T, et al. Association between radiation dose to neuronal progenitor cell niches and temporal lobes and performance on neuropsychological testing in children: a prospective study. Neuro Oncol. 2013;15:360-9.

26. Wiggenraad RG, Petoukhova AL, Versluis L, van Santvoort JP. Stereotactic radiotherapy of intracranial tumors: a comparison of intensity-modulated radiotherapy and dynamic conformal arc. Int J Radiat Oncol Biol Phys. 2009: 74:1018-26

27. Dolson EP, Conklin HM, Li C, Xiong X, Merchant TE. Predicting behavioral problems in craniopharyngioma survivors after conformal radiation therapy. Pediatr Blood Cancer. 2009:52:860-4.

28. Erfurth EM, Holmer H, Fjalldal SB. Mortality and morbidity in adult craniopharyngioma. Pituitary. 2013;16:46-55.

\section{Submit your next manuscript to BioMed Central and we will help you at every step:}

- We accept pre-submission inquiries

- Our selector tool helps you to find the most relevant journal

- We provide round the clock customer support

- Convenient online submission

- Thorough peer review

- Inclusion in PubMed and all major indexing services

- Maximum visibility for your research

Submit your manuscript at wuw biomedcentral.com/submit

) Biomed Central 